\title{
Tailoring Grain Boundary and Resultant Plasticity of Pure Iron by Pulsed-Electric-Current Treatment
}

\begin{abstract}
C. YANG, Y.J. ZHAO, Z. WANG, S.G. QU, X.Q. LI, W.W. ZHANG, and L.C. ZHANG
In general, annealing twins ( $\Sigma 3$ boundaries) are induced in face-centered cubic metals by thermal-mechanical processes. We report on the $\Sigma 3$ boundary-induced plasticity enhancement of pure iron treated by a pulsed electric current subject to its allotropic transformation of $\alpha \rightarrow \gamma \rightarrow \alpha$. The behavior is attributed to an increased amount of $\Sigma 3$ boundaries with the grain-boundary interconnection evolution from $\{112\} /\{112\}$ to $\{110\} /\{110\}$ resulting from the increasing pulsed-electric-current intensity. The results provide an alternative pathway to fabricate high-performance metallic materials by tailoring special grain boundaries.
\end{abstract}

https://doi.org/10.1007/s11661-018-5012-6

(C) The Minerals, Metals \& Materials Society and ASM International 2018

\section{INTRODUCTION}

As an important microstructural element, grain boundaries affect the mechanical, physical, electrical, and chemical properties of polycrystalline metals significantly. ${ }^{[1-3]} \mathrm{A}$ well-known example of this phenomenon is that coarse-grained polycrystalline metals are strengthened with the decreasing grain size, which implies the increasing total grain-boundary area, following the classical Hall-Petch relationship. ${ }^{[4]}$ Another successful example is that in grain-boundary engineering, related properties can be mediated by tailoring grain boundaries. ${ }^{5]}$ In such a case, a grain-boundary interconnection (GBIC), which is expressed as $\left\{h_{1} k_{l} l_{1}\right\} /$ $\left\{h_{2} k_{2} l_{2}\right\}$ by the Miller indices of the two interconnected crystallographic planes, is an appropriate approach to describe the characteristics of a grain boundary accurately. ${ }^{[6]}$ According to crystallography rules, GBIC dominates the microstructures that contain dislocations, free volume, and the faceting mode ${ }^{[7]}$ of a grain boundary. A manipulation of the distribution of characteristics for grain boundaries, ${ }^{[8]}$ which implies the

C. YANG and Y.J. ZHAO are with the Guangdong Key Laboratory for Advanced Metallic Materials Processing, South China University of Technology, Guangzhou 510640, China and also with the National Engineering Research Center of Near-NetShape Forming for Metallic Materials, South China University of Technology, Guangzhou 510640 China. Contact e-mail: cyang@scut.edu.cn Z. WANG is with the Guangdong Key Laboratory for Advanced Metallic Materials Processing, South China University of Technology. S.G. QU, X.Q. LI, and W.W. ZHANG are with the National Engineering Research Center of NearNet-Shape Forming for Metallic Materials, South China University of Technology. L.C. ZHANG is with the School of Engineering, Edith Cowan University, 270 Joondalup Drive, Joondalup, Perth, WA 6027 , Australia.

Manuscript submitted June 5, 2018.

Article published online November 19, 2018 increasing fraction of special low- $\Sigma(1 \leq \Sigma \leq 29)$ coincident-site-lattice (CSL) grain boundaries compared with random grain boundaries, can change the fracture mode, improve the mechanical properties of polycrystalline metals, and transform typical intergranular to transgranular fractures in Al-Li alloys, which results in its super-plasticity at elevated temperatures. ${ }^{[9]}$ Thus far, considerable endeavors have been made to anneal twin ( $\Sigma 3$ boundaries)-induced grain-boundary engineering, mainly in face-centered cubic (fcc) metals with a low stacking-fault energy. ${ }^{[10,11]}$ Technically, the method used in the current study is termed a thermal-mechanical process,${ }^{[12]}$ and includes a low-temperature deformation and a subsequent high-temperature annealing treatment. This scenario raises an interesting question: is there any processing method, other than the thermal-mechanical process, which can be introduced to tailor low- $\Sigma$ boundaries and the resultant mechanical properties of polycrystalline metals?

Recently, pulsed-electric-current (PEC) treatment was proposed as an effective method to improve the mechanical properties of polycrystalline metals given its ability to yield tailored microstructures (grain size and morphology). ${ }^{[13]}$ As one of the PEC treatment methods, spark-plasma-sintering, which couples an electric field that is induced by a PEC and thus a temperature field, with a pressure field ${ }^{[14]}$ can be applied to treat polycrystalline metals, and thus tailor texture and improve plasticity, ${ }^{[15]}$ except for its common application to consolidate powder particles and to achieve high-performance bulk materials. ${ }^{[16-18]}$ The underlying PEC mechanism is thought to be an accelerated nucleation of a crystallize phase and a suppression of grain growth under the coupled influence of thermal (temperature field) and athermal (facilitated atomic diffusion) effects. ${ }^{[19-22]}$ However, inspection of the published literature shows that tailoring the low- $\Sigma$ boundaries 
and the resultant mechanical properties of polycrystalline metals by a PEC has not been attempted previously.

We report on the $\Sigma 3$ boundary-induced plasticity enhancement of pure-iron that undergoes an allotropic transformation of $\alpha \rightarrow \gamma \rightarrow \alpha$ that is induced by a PEC that is generated in a spark-plasma-sintering system. To our knowledge, this is the first study that the polycrystalline metal plasticity has been improved by tailoring a special grain boundary from PEC treatment.

\section{EXPERIMENTAL}

The raw material was commercially cast pure-iron ingot (YT01). To refine the grains and to eliminate the texture or crystal orientation in the resultant microstructure, the cast pure-iron ingot was processed by cold rolling followed by annealing at $750{ }^{\circ} \mathrm{C}$ for 1 hour and final air cooling. The processed pure-iron billets had final dimensions of a $15-\mathrm{mm}$ diameter and a $120-\mathrm{mm}$ length. Samples $(15$-mm-diameter $\times 42$-mm-long rods $)$ from the billets were treated via PEC that was generated by a spark-plasma-sintering system (SPS-825, Sumitomo Coal Mining Co. Ltd., Japan) under the protection of an argon atmosphere. A schematic diagram of the mold assembly used for the PEC treatment is shown in Figure 1(a). The treated pure-iron rods were packed with graphite paper to allow the PEC to pass through the rods directly and to minimize the influence of any thermal stresses on the rods. The treatment parameters (Figure 1(b)) were designed as initial heating to $760{ }^{\circ} \mathrm{C}$ from room temperature; holding for 1.5 minutes; heating to $960{ }^{\circ} \mathrm{C}$, which far exceeds the body-centered cubic (bcc) $\alpha \leftrightarrow \gamma$ (fcc) transformation temperature of pure iron $\left(912{ }^{\circ} \mathrm{C}\right)$, at various heating rates of $50{ }^{\circ} \mathrm{C}, 100^{\circ} \mathrm{C}$, and $200^{\circ} \mathrm{C} / \mathrm{min}$, induced by varying maximum PEC intensities, i.e., 1400, 1600, and $1900 \mathrm{~A}$, respectively; and cooling to room temperature in the furnace chamber by switching off the electric supply at $960{ }^{\circ} \mathrm{C}$. No pressures and holding times were used in the experiments. The macroscopic temperature was controlled by a thermocouple close to the packed graphite paper. For data reproducibility, three runs for each PEC treatment parameter were conducted and characterized.

Cubic specimens $(6 \mathrm{~mm} \times 6 \mathrm{~mm} \times 6 \mathrm{~mm})$ were sectioned from the as-treated pure-iron rods and used for analyses. Surfaces X and Y on the as-treated cube specimens were perpendicular and parallel to the PEC direction (Figure 1(a)); only related results on surface X were presented in our case because of the isotropy on the two surfaces. Subsequently, cubic specimens were polished electrochemically by an electropolisher (EP-06, Shanghai Lab Testing Technology Co., Ltd.) and examined using electron backscattered diffraction (EBSD) in a Philips XL-30 FEG scanning electron microscope (SEM; Amsterdam, The Netherlands). The grain size, orientation imaging microscopy, grain-boundary misorientation, special CSL grain boundaries, ${ }^{[23]}$ orientation distribution function, and stereographic projections were determined by obtaining and analyzing the statistical relevance of the EBSD data of 10 mappings using HKL-Channel 5 software attached to the SEM. Tensile tests with a tensile direction parallel to the PEC direction (Figure 1(a)) were performed on the as-treated specimens at room temperature using a universal testing machine (Instron 5569) equipped with a laser extensometer. Tensile specimens with a 4-mm-diameter gauge section with a 16-mm length according to Chinese GB/T 228-2002 were tested at a strain rate of $0.033 \mathrm{~mm} / \mathrm{s}$.

\section{RESULTS}

Figure 2 shows the tensile true stress-strain curves of the initial and as-treated pure-iron specimens at various heating rates (maximum intensities of PEC) of $50^{\circ} \mathrm{C} /$

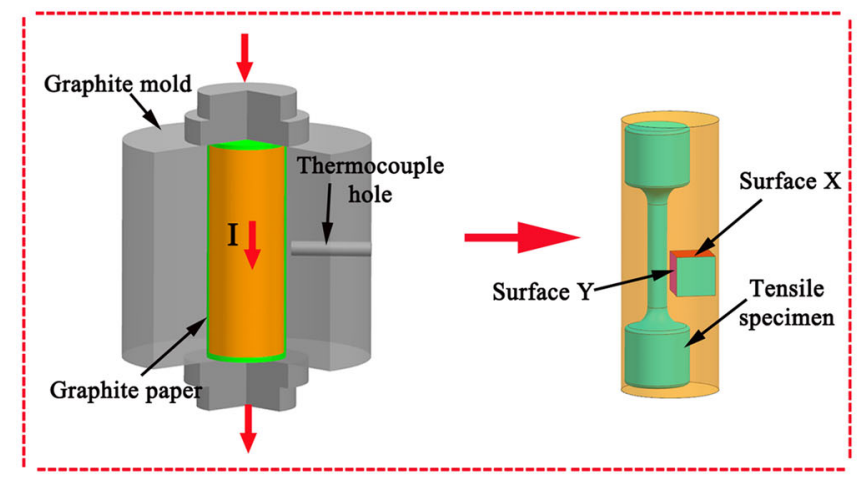

(a)

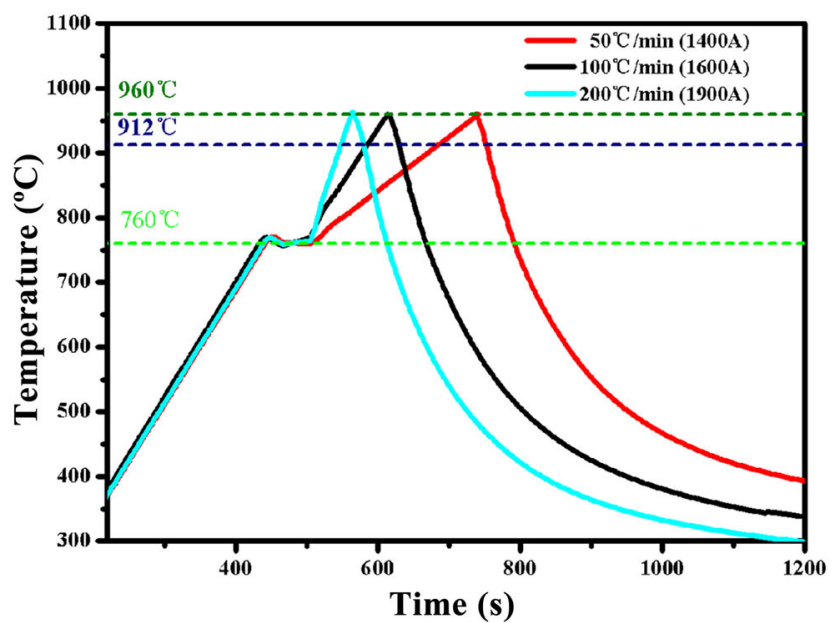

(b)

Fig. 1-(a) Schematic diagram of the mold assembly for PEC treatment and sampling-location maps of cubic and tensile specimens. (b) Temperature-time curves of PEC treatment. 
$\min (1400 \mathrm{~A}), 100^{\circ} \mathrm{C} / \min (1600 \mathrm{~A})$, and $200^{\circ} \mathrm{C} / \mathrm{min}$ (1900 A). The initial pure-iron specimen that was subjected to rolling and annealing had an ultimate tensile strength of $442 \pm 8 \mathrm{MPa}$ and a plastic strain of $21.5 \pm 1.2$ pct. In contrast, the as-treated specimens that were subjected to the extra PEC treatment exhibited

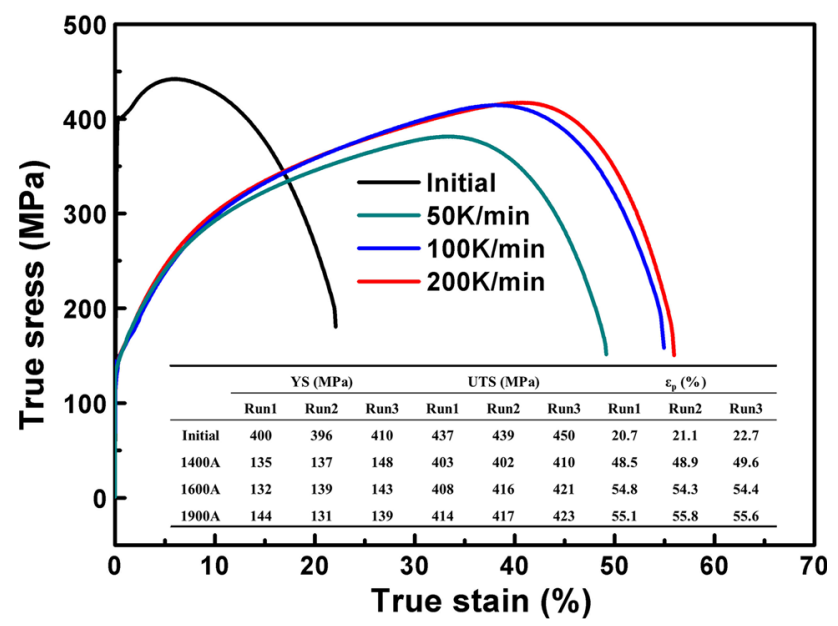

Fig. 2-Tensile true stress-strain curves for as-treated pure-iron specimens at various maximum PEC intensities of 1400, 1600, and $1900 \mathrm{~A}$. The inset lists correspond to the yield strength (YS), ultimate tensile strength (UTS), and plastic strain $\left(\varepsilon_{\mathrm{p}}\right)$.
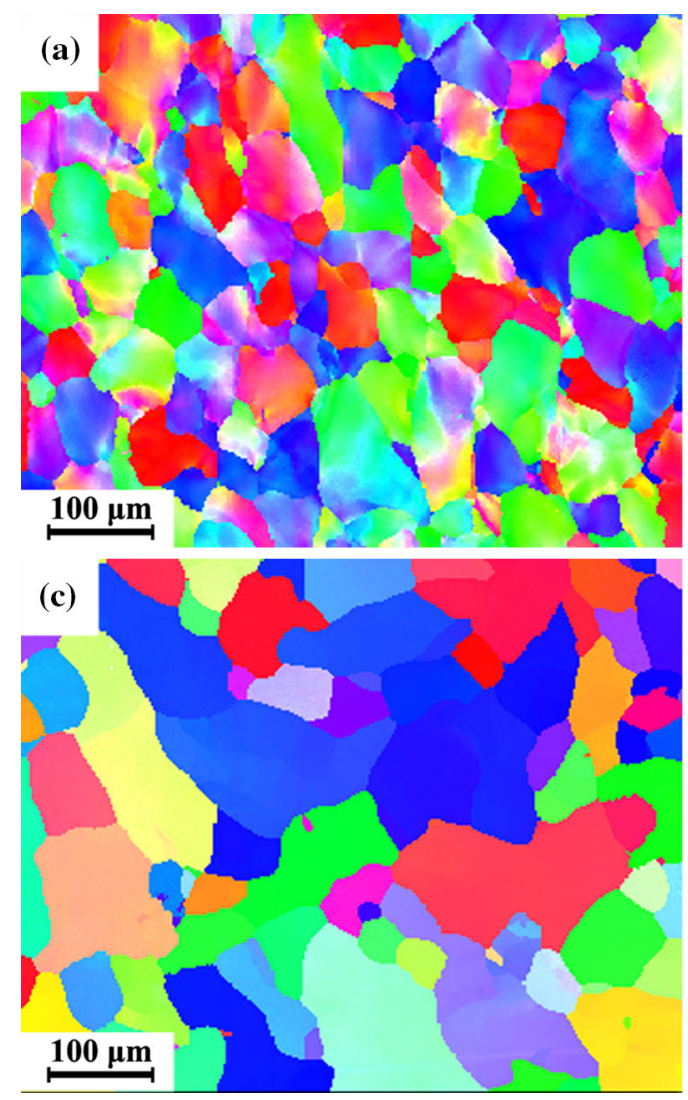

Fig. 3-Orientation imaging microscopy images on surface $\mathrm{X}$ in the initial $(a)$ and as-treated $(b-d)$ pure-iron specimens for various maximum PEC intensities of $1400(b), 1600(c)$, and $1900 \mathrm{~A}(d)$.
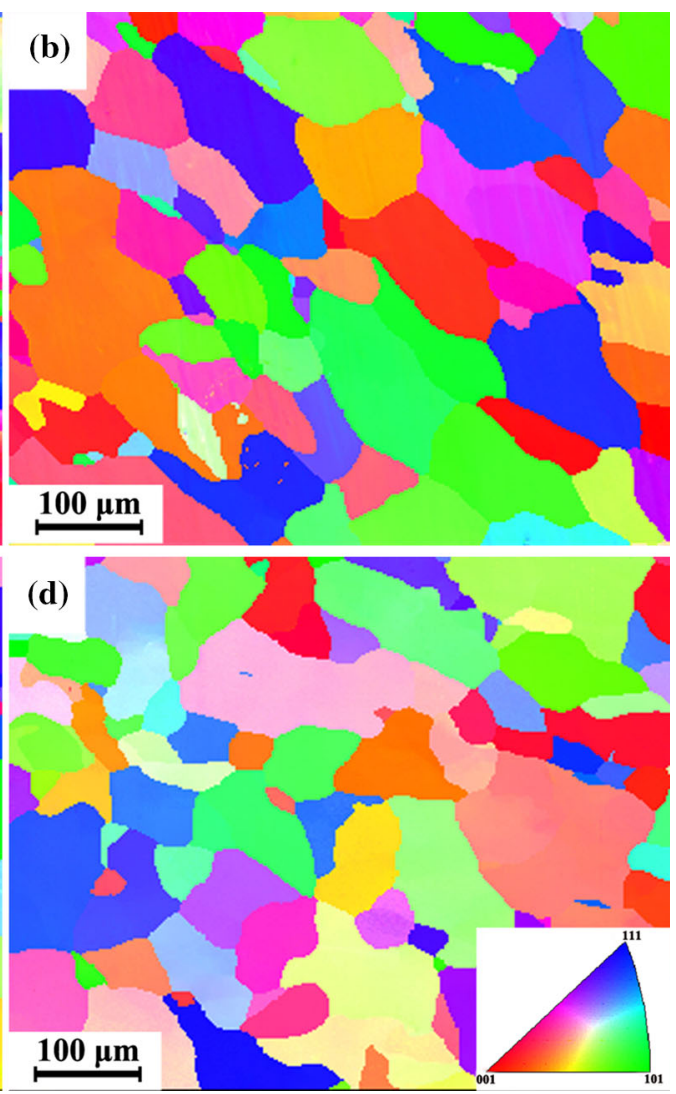

sharply decreased yield strengths, slightly decreased ultimate strengths, and significantly increased plastic strain. The sharp decrease in yield strength is attributed to rapidly increased grain sizes for the as-treated specimens, as presented in Figure 3. The plastic strain increases from $49.0 \pm 0.6$ to $54.5 \pm 0.3$ and $55.5 \pm$ 0.4 pct (Figure 2 inset), respectively, for the as-treated specimens with the increasing PEC intensity from 1400 to 1600 and $1900 \mathrm{~A}$, respectively.To distinguish the reason for the increased plastic strain with the increasing PEC intensity, Figure 3 presents orientation imaging microscopy images in the as-treated pure-iron specimens for various maximum PEC intensities of 1400, 1600, and 1900 A. Statistical analyses indicate that, compared with the average grain size of the initial specimen $(35.0 \mu \mathrm{m})$ in Figure 3(a), the corresponding grain sizes are $59.4 \mu \mathrm{m}$ (Figure 3(b)), $52.6 \mu \mathrm{m}$ (Figure 3(c)), and $45.9 \mu \mathrm{m}$ (Figure 3(d)), respectively, for the as-treated specimens at 1400, 1600, and $1900 \mathrm{~A}$, respectively. The as-treated specimens display a higher mean grain size than the initial sample. The grain size decreases significantly with the increasing PEC density. The result indicates that a higher PEC density can form a more homogeneous grain size. The grain refinement of the as-treated pure-iron specimens can be attributed to the coupled thermal and electrical effects that are induced by the PEC during the $\alpha \rightarrow \gamma$ transformation of pure iron. PEC treatment is a nonequilibrium process that is seen as a nonuniform rapid heating process, and can improve the 
nucleation rate. ${ }^{[24,25]}$ Theoretically, grain refinement in the as-treated pure-iron specimens by a high-intensity PEC during the $\alpha \rightarrow \gamma$ phase transformation can be expressed as ${ }^{[26]}$

$$
\begin{gathered}
\xi\left(\sigma_{1}, \sigma_{2}\right)=\left(\sigma_{2}-\sigma_{1}\right) /\left(\sigma_{2}+\sigma_{1}\right), \\
\Delta W_{e}=\mu_{0} \cdot g \cdot \xi\left(\sigma_{1}, \sigma_{2}\right) j^{2} \Delta V, \\
W_{\mathrm{c}}=\Delta W_{0}+\Delta W_{\mathrm{e}},
\end{gathered}
$$

where $\xi\left(\sigma_{1}, \sigma_{2}\right)$ is a factor that depends on the electrical properties of the $\alpha$ and $\gamma$ phases; and $\sigma_{1}$ and $\sigma_{2}$ are the conductivities of the $\alpha$ and $\gamma$ phases, respectively. $\Delta W_{\mathrm{e}}$ is the free-energy change that results from the change in distribution of the electric current during the formation of the $\gamma$ nucleus; $\mu_{0}$ is the magnetic susceptibility in a vacuum; $g$ is the positive geometric factor for coarse-grained materials; $j$ is the current intensity; and $\Delta V$ is the volume of a nucleus of the $\gamma$ phase. $W_{\mathrm{c}}$ is the free-energy change for the $\alpha \rightarrow \gamma$ phase transformation; and $\Delta W_{0}$ is the free-energy change in a current-free system. During the $\alpha \rightarrow \gamma$ phase transformation, because the packing density (68 pct) of the bcc crystal lattice in the $\alpha$ phase was lower than that $(74 \mathrm{pct})$ of the fcc crystal lattice in the $\gamma$ phase, $\sigma_{\gamma}$ $\left(=\sigma_{2}\right)$ was higher than $\sigma_{\alpha}\left(=\sigma_{1}\right)$, which leads to $\xi\left(\sigma_{1}, \sigma_{2}\right)>0$ according to Eq. [1] and $\Delta W_{\mathrm{e}}>0$ in Eq. [2]. The $\Delta W_{\mathrm{e}}$ increased with the increasing PEC intensity $(j)$ according to Eq. [2]. According to classical nucleation theory, only crystals with a critical radius can eventually develop into a nucleus of nucleation, and the average number of embryos with a critical radius is given by ${ }^{[27]}$

$$
n=n_{0}^{\prime} \exp \left(-W_{\mathrm{c}} / k T\right),
$$

where $n_{0}^{\prime}$ is the total number of atoms in the system; $k$ is the Boltzmann constant; and $T$ is the phase-transformation temperature. Therefore, the increasing PEC intensity could improve the nucleation rate in the $\alpha \rightarrow \gamma$ phase transformation significantly by decreasing the thermodynamic barrier. ${ }^{[28]}$ For a rapid phase change, because the newly formed phase does not have sufficient time to merge and grow, the final grain size of the new phase depends mainly on the nucleation rate. Thus, the grain size decreases with the increasing PEC intensity.

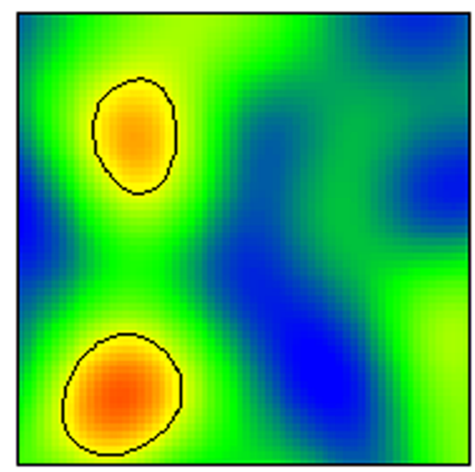

$\phi 2=45^{\circ}$

(a)

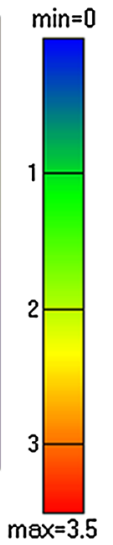

$\max =3.5$

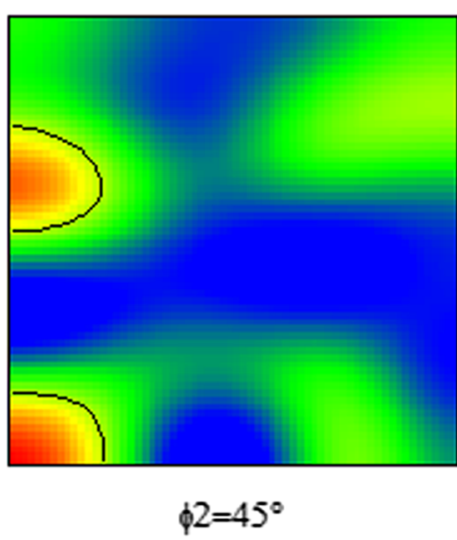

(b)
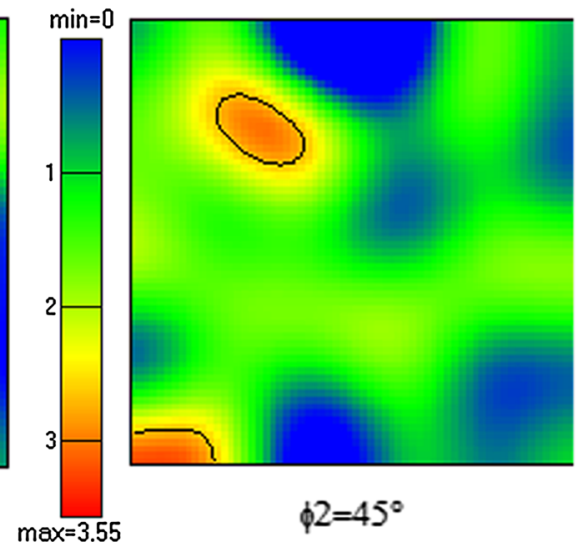

(c)

Fig. 4 - Orientation distribution function of as-treated pure-iron specimens at various maximum PEC intensities of: (a) 1400, (b) 1600, and (c) 1900 A.

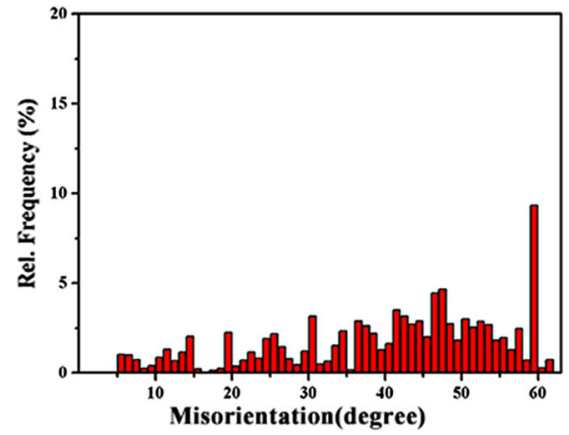

(a)

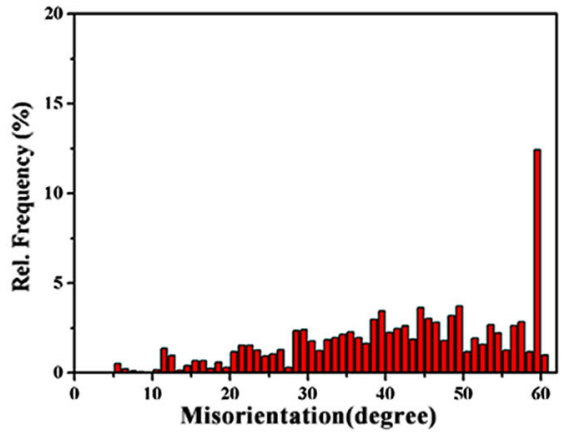

(b)

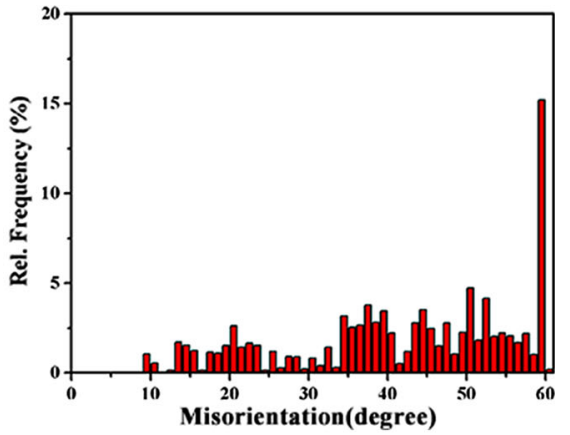

(c)

Fig. 5-Misorientation distributions for as-treated pure-iron specimens by various maximum PEC intensities of (a) 1400, (b) 1600, and (c) 1900 A. 
Table I. Frequency of the $\Sigma 3$ Boundaries, Low- $\Sigma$ Boundaries, HABs in the As-treated Pure-Iron Samples

\begin{tabular}{|c|c|c|c|c|c|c|c|c|c|}
\hline \multirow[b]{2}{*}{ PEC Intensity } & \multicolumn{3}{|c|}{$\Sigma 3$ Boundaries Frequency (Percent) } & \multicolumn{3}{|c|}{ Low- $\Sigma$ Boundaries Frequency (Percent) } & \multicolumn{3}{|c|}{ HABs (Percent) } \\
\hline & Run 1 & Run 2 & Run 3 & Run 1 & Run 2 & Run 3 & Run 1 & Run 2 & Run 3 \\
\hline $1400 \mathrm{~A}$ & 9.6 & 9.3 & 8.8 & 19.3 & 18.7 & 18.5 & 90.5 & 98.1 & 95.1 \\
\hline $1600 \mathrm{~A}$ & 13.4 & 13.2 & 13.1 & 22.3 & 21.4 & 22.8 & 96.0 & 99.1 & 97.4 \\
\hline $1900 \mathrm{~A}$ & 15.5 & 15.1 & 15.3 & 25.8 & 25.7 & 25.0 & 95.5 & 99.6 & 99.4 \\
\hline
\end{tabular}

Figure 3 shows that there is no significant difference in crystallographic texture for the as-treated specimens. The same treatment temperature and cooling procedure used in our case (Figure 1(b)) can exclude the effect of cooling rate on microstructural evolution in the allotropic transformation $\mathrm{fcc} \rightarrow \mathrm{bcc}$ of the as-treated specimens. Therefore, the decreased grain size with the increasing PEC intensity can be attributed to an acceleration of nucleation and the suppression of grain growth during the allotropic transformation bcc $\rightarrow$ fcc of the as-treated specimens, which results from the coupled influence of PEC thermal and athermal effects. ${ }^{[2]}$ Two different phenomena exist for the as-treated pure-iron specimens compared with the PEC-treated pure titanium under similar treatment conditions, ${ }^{[15]}$ i.e., their allotropic transformation temperatures are exceeded. The change in grain size with the increasing PEC intensity is inversely correlated to that for PEC-treated pure titanium. The texture evolution in the as-treated specimens does not exhibit a regularity with the increasing PEC intensity together with relative low intensities (Figures 3(b) through (d) and 4(a) through (c)), which is different from the significantly weakened texture for PEC-treated pure titanium. In general, when the allotropic transformation temperatures are exceeded, the resultant iron and titanium experience two phase transformations: bcc $\rightarrow$ fcc and subsequent fcc $\rightarrow$ bcc; and hexagonal closepacked (hcp) $\rightarrow$ bcc and subsequent bcc $\rightarrow$ hcp, respectively. Unlike pure titanium that is subjected to an allotropic transformation with different crystallographic systems, the as-treated pure-iron specimens experience an allotropic transformation with the same crystallographic systems. This may lead to no prominent texture evolution for the as-treated pure-iron specimens with PEC intensity. Similar results have been reported in microalloyed steel. $^{[30]}$ Commonly, the plasticity of polycrystalline metals decreases with the decreasing grain size according to the Hall-Petch relationship. ${ }^{[4]}$ The increased plastic strain (Figure 2) with the decreasing grain size (Figure 3) for the as-treated specimens may originate from other underlying crystallographic reasons, such as the grain-boundary character distribution.

To justify this deduction, Figure 5 shows the misorientation distributions and fractions of $\Sigma 3$ boundaries and low- $\Sigma$ boundaries for the as-treated pure-iron specimens at various maximum PEC intensities of 1400,1600 , and $1900 \mathrm{~A}$. The frequency of the $\Sigma 3$ boundaries, low- $\Sigma$ boundaries, and high-angle grain boundaries (HABs) in the as-treated pure iron are listed in Table I. The as-treated specimens have a vast majority of HABs with a misorientation higher than
$15 \mathrm{deg}$ (Figures 5(a) through (c) and Table I) because of the formation of new large grains (Figures 3(b) through (d). The $\Sigma 3$ boundaries $(60 \mathrm{deg}\langle 111\rangle)$ that correspond to a misorientation at $60 \mathrm{deg}$ increase from $9.2 \pm 0.4$ to $13.2 \pm 0.2$ and to $15.3 \pm 0.2 \mathrm{pct}$ for the as-treated specimens with the increasing PEC intensity from $1400 \mathrm{~A}$ to $1600 \mathrm{~A}$ and further to $1900 \mathrm{~A}$ (Table I). Concomitantly, the frequency of the low- $\Sigma$ boundaries increases with the increasing PEC intensity.

\section{DISCUSSION}

It has been reported that the introduction of a high fraction of special low- $\Sigma$ (especially $\Sigma 3$ ) boundaries by thermal-mechanical processing can contribute to a high structural-order degree and regulate the fracture mode, which improves the mechanical properties of the polycrystalline metals. ${ }^{[9,31]}$ Therefore, the increased plastic strain with the increasing PEC intensity (Figure 2) can be rationalized as increased low- $\Sigma$ (especially $\Sigma$ 3) boundaries (Figure 5).

To authenticate this rationalization, the types of GBICs of $\Sigma 3$ boundaries in the as-treated pure-iron specimens were measured and determined based on statistical analysis. The measurement of GBICs $\left\{h_{1} k_{1} l_{1}\right\} /\left\{h_{2} k_{2} l_{2}\right\}$ of grain boundaries was accomplished by measuring the grain-boundary plane orientation assisted by crystallographic analysis at a specific misorientation. The intensity of the grain-boundary plane orientation in a certain orientation can be determined by the function $\lambda(\Delta g, n)$ in which $\Delta g$ is the misorientation between the two adjacent grains, and $n$ is the orientation of the grain-boundary plane in the crystallographic coordinate. ${ }^{[32,33]}$ The distributions of all grain-boundary plane orientations in the top semisphere of the crystallographic space is determined, and the distribution intensity in each orientation bin is a multiple of random distribution (MRD). Usually, the $\lambda(\Delta g, n)$ of cubic materials is projected onto a specific crystallographic plane, such as $\left(\begin{array}{lll}0 & 0 & 1\end{array}\right)$. Based on the $\lambda(\Delta g$, n) results of the $\Sigma 3$ boundaries with a $60 \mathrm{deg} /\left[\begin{array}{lll}1 & 1 & 1\end{array}\right]$ misorientation, the GBICs of this type of boundary can be determined from ${ }^{[34]}$ :

$$
\left[\begin{array}{c}
h 2 \\
k 2 \\
l 2
\end{array}\right]=\left[\begin{array}{ccc}
2 & -1 & 2 \\
2 & 2 & -1 \\
-1 & 2 & 2
\end{array}\right]\left[\begin{array}{c}
h 1 \\
k 1 \\
l 1
\end{array}\right]
$$

Figure 6 shows the (001) stereographic projections of the grain-boundary plane orientations of $\Sigma 3$ boundaries 


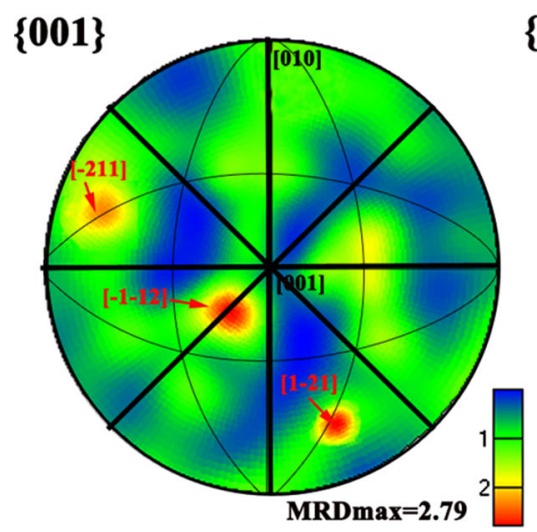

(a)
$\{001\}$

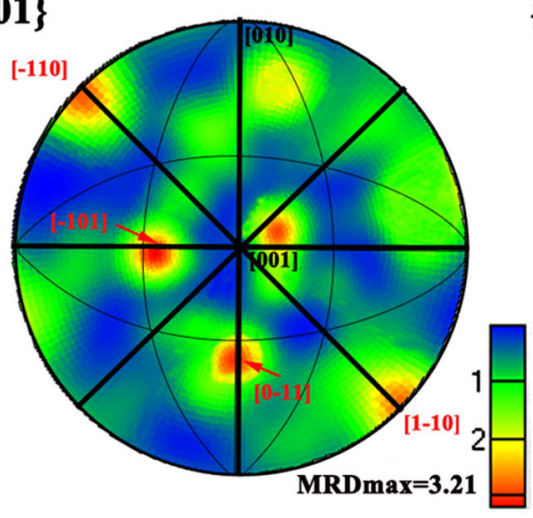

(b)

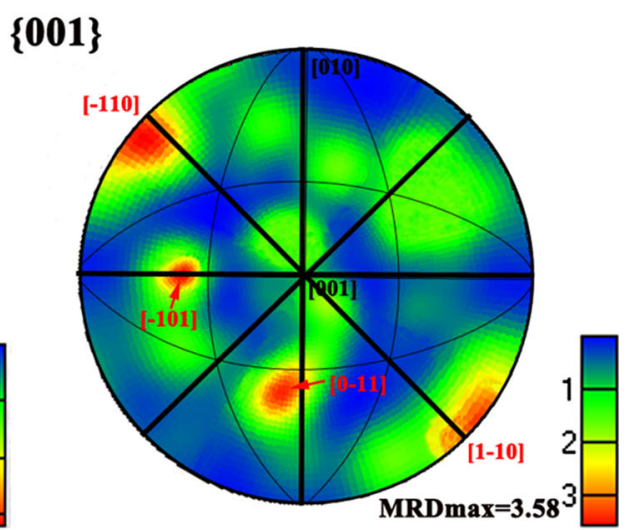

(c)

Fig. 6-(001) stereographic projections of grain-boundary plane orientations of $\Sigma 3$ boundaries in as-treated pure-iron specimens by various maximum PEC intensities of $(a)$ 1400, (b) 1600, and (c) $1900 \mathrm{~A}$.

in the as-treated pure-iron specimens by various maximum PEC intensities of 1400, 1600, and $1900 \mathrm{~A}$. Figure 6 and Eq. [1] show that the GBIC of $\Sigma 3$ boundaries in the 1400 A-treated specimen is $\{112\} /$ $\{112\}$. In contrast, with the increasing PEC intensity to 1600 and $1900 \mathrm{~A}$, the GBICs of the $\Sigma 3$ boundaries transform into $\{110\} /\{110\}$, together with slightly stronger grain-boundary plane orientations. This indicates that the GBIC of the $\Sigma 3$ boundaries pure-iron specimens tends to the low-index planes that undergo a reversible allotropic transformation of bcc $\rightarrow \mathrm{fcc}$ and subsequent $\mathrm{fcc} \rightarrow$ bcc with the increasing PEC intensity. The MRD maximum-intensity values of the stereographic projections increased with the increasing heating rates (PEC intensities) (Figure 6), which suggests a gradual enhancement of preferred crystal orientation. The evolution of GBIC with PEC intensity is consistent with the scenario in pure iron that is subjected to thermal-mechanical processing, ${ }^{[30]}$ and verifies that the $\{110\} /\{110\}$ is the most stable GBIC for pure iron. This case can be explained based on the following two factors. According to the theories of CSL, ${ }^{[22]}$ the planar coincident-site densities on the boundary plane are $10.31 \mathrm{~nm}^{-2}$ and $18.05 \mathrm{~nm}^{-2}$ for the $\{112\} /\{112\}$ and $\{110\} /\{110\}$ GBICs, respectively. The degree of lattice mismatch is inversely correlated with the values of the planar coincident-site densities. ${ }^{[35]}$ Extensive data have confirmed that low-index planes with low surface energies often exist in low-energy grain boundaries. ${ }^{[36,37]}$ Consequently, grain boundaries that are terminated by the (110) planes have relatively low energies for bcc iron compared with other boundaries. The effect of PEC can be speculated as a possible transformation of polycrystalline metals, and a higher packing density of crystal lattice. ${ }^{[16,38]}$ This agrees well with the aforementioned two factors. The (110) planes are the easiest slip plane of bcc iron. This implies that the $\{110\} /\{110\}$ GBIC can facilitate dislocation and slip movement between two interconnected crystallographic planes, i.e., two interconnected grains. Spontaneously, the evolution of GBIC from $\{112\} /\{112\}$ to $\{110\} /\{110\}$ (Figure 6) and the increased fraction of $\Sigma 3$ boundaries (Figures 3 and 5 and Table I) give rise to a plasticity enhancement (Figure 2) with the increasing PEC intensity for the as-treated pure-iron specimens.

\section{CONCLUSIONS}

$\Sigma 3$ boundary-induced plasticity enhancement of pure iron was achieved by PEC treatment with its allotropic transformation of $\alpha \rightarrow \gamma \rightarrow \alpha$. The plastic strain of the as-treated pure-iron specimens increased with the increasing PEC intensity. This behavior is rationalized as an increased fraction of $\Sigma 3$ boundaries and GBIC evolution from $\{112\} /\{112\}$ to $\{110\} /\{110\}$ with the increasing PEC intensity. The results confirm that PEC treatment is an alternative pathway for fabricating high-performance metallic materials.

\section{ACKNOWLEDGMENTS}

The current study was supported by the National Natural Science Foundation of China (No. 51574128), the Guangdong Natural Science Foundation for Research Team (No. 2015A030312003), the Guangdong Application-oriented Special Funds for Science and Technology R\&D (No. 2016B090931002), and the Fundamental Research Funds for the Central Universities (No. 2017PY014).

\section{REFERENCES}

1. S.E. Babcock, X.Y. Cai, D.L. Kaiser, and D.C. Larbalestier: Nature, 1990, vol. 347, pp. 167-69.

2. A. Sutton and R. Balluffi: Interfaces in Crystalline Materials, Oxford Unverisity Press, Oxford, 1995.

3. D. Brandon: Mater. Sci. Technol, 2010, vol. 26, pp. 762-73.

4. T.N. Baker: Yield Flow and Fracture of Polycrystals, Applied Science Publishers, London, 1983.

5. S. Yamaura, Y. Igarashi, S. Tsurekawa, and T. Watanabe: Acta Mater., 1999, vol. 47, pp. 1163-74.

6. D. Wolf, and S. Yip: Chapman \& Hall, London, 1992. 
7. E. Tochigi, Y. Kezuka, N. Shibata, A. Nakamura, and Y. Ikuhara: Acta Mater., 2012, vol. 60, pp. 1293-99.

8. P. Lin, G. Palumbo, U. Erb, and K.T. Aust: Scr. Metall. Et Materialia, 1995, vol. 33, pp. 1387-92.

9. T. Watanabe and S. Tsurekawa: Acta Mater., 1999, vol. 47, pp. 4171-85.

10. S. Xia, B.X. Zhou, W.J. Chen, and W.G. Wang: Scr. Mater., 2006, vol. 54, pp. 2019-22.

11. V. Randle: J. Mater. Sci., 2005, vol. 40, pp. 853-59.

12. N.Y. Zolotorevsky, N.Y. Ermakova, V.S. Sizova, E.A. Ushanova, and V.V. Rybin: J. Mater. Sci., 2017, vol. 52, pp. 4172-81.

13. J.G. Santanach, A. Weibel, C. Estournes, Q. Yang, C. Laurent, and A. Peigney: Acta Mater., 2011, vol. 59, pp. 1400-08.

14. Z.A. Munir, U. Anselmi-Tamburini, and M. Ohyanagi: J. Mater. Sci., 2006, vol. 41, pp. 763-77.

15. C. Yang, J.A. Lin, Y.F. Ding, W.W. Zhang, Y.Y. Li, Z.Q. Fu, F. Chen, and E.J. Lavernia: J. Mater. Sci., 2016, vol. 51, pp. 1-12.

16. C. Yang, L.M. Kang, X.X. Li, W.W. Zhang, D.T. Zhang, Z.Q. Fu, Y.Y. Li, L.C. Zhang, and E.J. Lavernia: Acta Mater., 2017, vol. 132, pp. 491-502.

17. L.H. Liu, C. Yang, F. Wang, S.G. Qu, X.Q. Li, W.W. Zhang, Y.Y. Li, and L.C. Zhang: Mater. Des., 2015, vol. 79, pp. 1-5.

18. C. Yang, Y.J. Zhao, L.M. Kang, D.D. Li, W.W. Zhang, and L.C. Zhang: Mater. Lett., 2018, vol. 210, pp. 169-72.

19. R. Li, T. Yuan, X. Liu, and K. Zhou: Scr. Mater., 2016, vol. 110, pp. $105-08$.

20. S. Deng, R. Li, T. Yuan, S. Xie, M. Zhang, K. Zhou, and P. Cao: Scr. Mater., 2018, vol. 143, pp. 25-29.

21. C. Yang, D.G. Mo, H.Z. Lu, X.Q. Li, W.W. Zhang, Z.Q. Fu, L.C. Zhang, and E.J. Lavernia: Scr. Mater., 2017, vol. 134, pp. 91-94.

22. X.X. Li, C. Yang, T. Chen, Z.Q. Fu, Y.Y. Li, O.M. Ivasishin, and E.J. Lavernia: Scr. Mater., 2018, vol. 151, pp. 47-52.
23. D.G. Brandon: Acta Mater., 1996, vol. 14, pp. 1479-84.

24. C. Antonione, G.G. Della, G. Riontino, and G. Venturello: $J$. Mater. Sci., 1973, vol. 8, pp. 1-10.

25. R. Song, D. Ponge, D. Raabe, J. Speer, and D. Matlock: Mater. Sci. Eng. A., 2006, vol. 441, pp. 1-17.

26. K.K. Surthi, R.K. Khatirkar, and S.G. Sapate: ISIJ Int., 2013, vol. 53, pp. 356-64.

27. I.L. Dillamore, C.J.E. Smith, and T.W. Watson: Metal Sci. J., 2013, vol. 1, pp. 49-54.

28. A. Borbély, J. Driver, and T. Ungár: Acta Mater., 2000, vol. 48, pp. 2005-16.

29. Y.B. Jiang, G.Y. Tang, C. Shek, Y.H. Zhu, and Z.H. Xu: Acta Mater., 2009, vol. 57, pp. 4797-4808.

30. I. Lischewski and G. Gottstein: Acta Mater., 2011, vol. 59, pp. $1530-41$.

31. T. Watanabe and S. Tsurekawa: Mater. Sci. Eng. A., 2004, vols. 387-389, pp. 447-55.

32. D.M. Saylor, B.E. Dasher, B.L. Adams, and G.S. Rohrer: Metall. Mater. Trans. A., 2004, vol. A35, pp. 1981-89.

33. W.G. Wang, Y. Lin, P. Dai, G.S. Rohrer, and W.Z. Zhang: Mater. Charact., 2016, vol. 122, pp. 6-13.

34. Y.H. Rong: Fatigue Strength, Higher Education Press, Beijing, 2006.

35. W.G. Wang, S. Chen, G.S. Rohrer, and W.Z. Chen: Scr. Mater., 2017, vol. 128, pp. 18-22.

36. J.M. Zhang, D.D. Wang, and K.W. Xu: Appl. Surf. Sci., 2006, vol. 252, pp. 8217-22.

37. S. Ratanaphan, D.L. Olmsted, V.V. Bulatov, E.A. Holm, A.D. Rollett, and G.S. Rohrer: Acta Mater., 2015, vol. 88, pp. $346-54$.

38. W. Chen, U. Anselmi-Tamburini, J.E. Garay, J.R. Groza, and Z.A. Munir: Mater. Sci. Eng. A., 2005, vol. 394, pp. 132-38. 